\title{
Investigating the Neural Correlates of Schemas: Ventromedial Prefrontal Cortex Is Necessary for Normal Schematic Influence on Memory
}

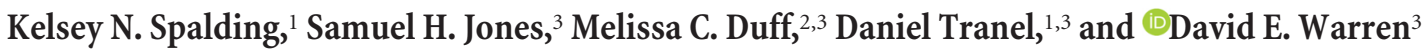 \\ Departments of ${ }^{1}$ Psychological and Brain Sciences and ${ }^{2}$ Communication Sciences and Disorders, College of Liberal Arts and Sciences, and ${ }^{3}$ Department of \\ Neurology, Carver College of Medicine, University of Iowa, Iowa City, Iowa 52242
}

\begin{abstract}
Schemas, as memory representations of typical contexts, allow for generalization from previous experiences while often improving memory organization and accuracy. However, these advantageous characteristics of schematic memory may come at the cost of episodespecific information. In the human brain, this tradeoff between general and specific knowledge has been linked to differential contributions of the medial temporal lobes (MTL) to episode-specific memory and the ventromedial prefrontal cortex (vmPFC) to generalized, schematic memory. Here, we used a neuropsychological approach to test whether participants with focal vmPFC damage $(n=6)$ would show a reduced influence of schematic memory relative to healthy normal comparison participants $(n=12)$ in a recognition task that presented schematically congruent or incongruent contexts at study. As predicted, normal comparison participants were more likely to identify items as old after studying them in congruent contexts, and this effect was reflected in increased true and false recognition. These effects of prior context on recognition were not observed in the vmPFC group, suggesting that vmPFC damage reduced the influence of schematic memory. These findings are consistent with the proposition that the vmPFC plays an important role in integrating previous experience into ongoing memory processes while acting as part of a larger memory network.
\end{abstract}

Key words: contextual congruence; false recognition; schema; schematic memory; ventromedial prefrontal cortex; vmPFC

Significance Statement

In the human brain, new memories are strongly influenced by existing knowledge of relevant context (sometimes called "schemas"). Schemas can benefit memory by expediting learning and increasing capacity in familiar contexts, but these benefits may simultaneously reduce episode-specific memory. Here we show that damage to the human ventromedial prefrontal cortex (vmPFC) reduced the influence of existing knowledge on new memories. Our findings suggest that the vmPFC plays a key role in schematic memory processes by integrating previous experiences and contextual information to influence memory. These findings provide novel insight into the brain regions necessary for normal schematic memory and enhance our understanding of the brain networks supporting memory processes.

\section{Introduction}

“... as beautiful as the chance meeting on a dissecting-table of a sewing-machine and an umbrella."

— Isidore Ducasse, Latréamont's Maldoror.

We can appreciate the bizarre juxtaposition of incongruent concepts by artists such as M. Ducasse because our memory systems

Author contributions: K.N.S., S.H.J., M.C.D., D.T., and D.E.W. designed research; K.N.S., S.H.J., and D.E.W. performed research; K.N.S. and D.E.W. analyzed data; K.N.S., S.H.J., M.C.D., D.T., and D.E.W. wrote the paper.

This work was supported by National Institute of General Medical Sciences Grant T32 GM108540 (K.N.S.), McDonnell Foundation UHC-Collaboration 220020387 (D.T.), National Institute on Deafness and Other Communication Disorders Grant R01 DC011755 (M.C.D.), and National Institute of Mental Health Grant R01 MH062500 (D.E.W.).

The authors declare no competing financial interests. constantly compare new experiences with contextual knowledge. The contextual knowledge supporting this comparison process has been described using the concept of schemas (Bartlett, 1932), memory structures hypothesized to be extracted over multiple exposures that are activated by the presence of related concepts. Schemas exert profound, frequently advantageous effects on memory organization and accuracy by expediting learning and expanding memory capacity (Morris, 2006; Tse et al., 2007; Wang and Morris, 2010; Lewis and Durrant, 2011). However, schematic effects can also be disadvantageous, as when proactive

Correspondence should be addressed to David E. Warren, 2155-H RCP, 200 Hawkins Drive, lowa City, IA 52242. E-mail: davideugenewarren@gmail.com.

DOI:10.1523/JNEUROSCI.2767-15.2015

Copyright $\odot 2015$ the authors $\quad 0270-6474 / 15 / 3515746-06 \$ 15.00 / 0$ 
Table 1. Demographic and neuropsychological information for the vmPFC participant group

\begin{tabular}{|c|c|c|c|c|c|c|c|c|c|c|c|c|c|c|c|c|c|c|c|c|}
\hline \multirow[b]{2}{*}{ ID } & \multirow[b]{2}{*}{ Age } & \multirow[b]{2}{*}{ Sex } & \multirow[b]{2}{*}{ Hand } & \multirow[b]{2}{*}{ Edu } & \multirow[b]{2}{*}{ Eti. } & \multirow[b]{2}{*}{ Chron } & \multicolumn{4}{|l|}{ WAIS } & \multirow[b]{2}{*}{ COWA } & \multicolumn{2}{|l|}{$C F$} & \multirow{2}{*}{$\frac{\text { AVLT }}{1 / 5 / \text { Delay }}$} & \multicolumn{4}{|l|}{ WMS } & \multicolumn{2}{|c|}{ Lesion volume } \\
\hline & & & & & & & VIQ & Sim & $\operatorname{lnf}$ & Voc & & Raw & Norm & & GMI & $\mathrm{Al}$ & $A D$ & ADR & Total & vmPFC \\
\hline 318 & 75 & M & 100 & 14 & Res. & 39 & 142 & 18 & 16 & 16 & 54 & 53 & 0.49 & $10 / 14 / 10$ & 109 & 118 & 114 & 125 & 76482 & 0.40 \\
\hline 2352 & 66 & $\mathrm{~F}$ & 100 & 14 & SAH & 14 & 108 & 11 & 11 & 10 & 34 & 54 & 0.61 & 7/14/11 & 109 & 114 & 111 & 115 & 11239 & 0.20 \\
\hline 2391 & 68 & $\mathrm{~F}$ & 100 & 13 & Res. & 13 & 110 & 12 & 12 & 12 & 59 & 49 & 0.01 & $11 / 15 / 14$ & 132 & 120 & 124 & 120 & 53719 & 0.56 \\
\hline 3350 & 63 & M & 100 & 18 & Res. & 11 & 119 & 14 & 12 & 11 & 40 & 39 & -1.92 & $8 / 14 / 13$ & 108 & 117 & 114 & 100 & 34082 & 0.50 \\
\hline 3534 & 75 & $\mathrm{~F}$ & 100 & 12 & Res. & 5 & $107^{a}$ & $14^{a}$ & $9^{a}$ & $11^{a}$ & 32 & 55 & 0.74 & $7 / 15 / 12$ & 117 & 134 & 114 & 135 & 34955 & 0.52 \\
\hline
\end{tabular}

vmPFC participants were cognitively intact, with little evidence of impairment in verbal functions or memory. ID, Patient identification; Hand, handedness, 100 (fully right-handed); Edu, education in years; Eti, etiology, including resection (res.), stroke (str.), subarachnoid hemorrhage (SAH); Chr, chronicity, years between brain injury and experiment; WAIS, Wechsler Adult Intelligence Scale, 3rd edition; VIQ, verbal IQ; Sim, Inf, and Voc, similarities, information, and vocabulary subtests age-corrected scaled scores; COWA, Controlled Oral Word Association task scaled scores; CF, category fluency task, raw score and age-corrected Z score; AVLT, Rey Auditory Verbal Learning Test, trial 1 performance, trial 5 performance, and 30 min delay performance; WMS, Wechsler Memory Scale-III; GMI, WMS general memory index; AI, WMS auditory immediate index; AD, WMS auditory delay index; ADR, WMS auditory delayed recognition index; Lesion volume, Total, total lesion volume $\left(\mathrm{mm}^{3}\right)$; vmPFC, proportion of vmPFC gray matter affected by lesion. Here, the vmPFC was an anatomically defined region of interest (Kumaran et al., 2015).

${ }^{a}$ WAIS-IV used for patient 3534.

interference causes recall of a typical experience instead of a specific, nontypical experience [e.g., incorrectly remembering that you spoke to a colleague at work (typical) rather than at a party (nontypical)]. Although psychologists have studied schematic memory processes for decades (for review, see Koriat et al., 2000; van Kesteren et al., 2012), the neural correlates of these processes are not well understood.

Investigations of schematic memory using cognitive neuroscience methods have offered preliminary evidence for involvement of the medial prefrontal cortex ( $\mathrm{mPFC}$ ) and perhaps more specifically the ventromedial PFC (vmPFC). Correlational evidence for vmPFC contributions to schematic memory has been provided by functional neuroimaging studies reporting increased activation in the human vmPFC when participants integrated conceptual knowledge or schematic memories (Kumaran et al., 2009; van Kesteren et al., 2010, 2013; Bonnici et al., 2012; Zeithamova et al., 2012a,b). Additionally, neuroimaging investigations by van Kesteren et al. (2010) showed increased mPFC activation and functional connectivity for schematically congruent stimuli at retrieval. This work prompted the hypothesis that the mPFC may support the generalization (or "schematization") of memories by identifying elements of experience that are schematically consistent during both encoding and retrieval (van Kesteren et al., 2012).

Neuropsychological evidence for vmPFC contributions to schematic memory processes has shown that bilateral vmPFC damage is associated with reduced susceptibility to schematic false recall using the Deese-Roediger-McDermott memory paradigm (Warren et al., 2014; but see Melo et al., 1999). These findings are broadly consistent with a large body of work demonstrating that the vmPFC plays an important role in integrating previous experiences to influence current decisionmaking (Bechara and Damasio, 2005). Together, the evidence suggests that the vmPFC may contribute to schematic memory processes by incorporating previous contextual knowledge into memory retrieval. A recent account incorporating contemporary findings has suggested that the vmPFC promotes schematic generalization from previous experience at the expense of specific information about the current experience (van Kesteren et al., 2012) leading to memory errors such as false recognition and false recall. However, the necessity of the vmPFC for normal schematic memory processes remains to be established.

In particular, additional investigation is needed to determine whether the vmPFC is necessary for other aspects of schematic memory, such as the perceived match between an object and its context (contextual congruence). Schemas strongly influence our contextual predictions by shaping rad- ically different expectations for, as an example, objects that might be found in a desert versus an ice rink (Palmer, 1975). We used a neuropsychological approach to assess whether the vmPFC is necessary for incorporating contextual congruence, evaluating the online and remembered influence of schematically congruent or incongruent contexts in healthy individuals and in patients with focal brain lesions including the vmPFC. We hypothesized that contextual congruence would modulate memory performance for normal comparison participants by promoting generalization from previous experience but that this schematic influence would be reduced in individuals with vmPFC damage.

\section{Materials and Methods}

\section{Participants}

Participants with focal, stable, bilateral vmPFC lesions ("vmPFC participants"; $n=6,2$ males and 4 females) were recruited from the Iowa Neurological Patient Registry. Neuropsychological testing was performed at least 3 months after lesion (Table 1). Lesions were verified using computerized tomography or magnetic resonance imaging and traced in a template space using the MAP3 lesion method (Frank et al., 1997). Maximum lesion overlap was found in the vmPFC (Fig. 1A). Healthy normal comparison participants ("normal comparisons" or "NC"; $n=12,4$ males and 8 females) were matched to the vmPFC participants (2:1) on sex, age, and education. The groups did not differ demographically (each $t_{(16)}<0.87$, each $p>0.399$ ). All participants provided informed consent in accordance with the Declaration of Helsinki. Participation was remunerated.

\section{Materials and Design}

The task used visual stimuli including context words and images of common objects. Objects and context words were combined into display items in a congruence-judgment phase. At test, objects were presented without context (see below, Procedure).

Object image stimuli were drawn from freely available materials (Yassa et al., 2011) including 384 objects, each represented with two exemplars that were visually similar (Fig. $1 B$ ). The authors generated context words for each object. Object-context congruence ratings were obtained from a pilot study with young adults $(n=11)$, and context assignments were finalized. Normative (in)congruence of the final object-context pairings was confirmed in another pilot study with young adults $(n=20)$.

Objects were assigned to experimental conditions based on a $3 \times 2$ design crossing recognition condition with contextual congruence. In each session, an equal number of objects ( 96 each) were assigned to one of three recognition conditions: old, similar, and new. Old and similar objects were split evenly (48 each) across two contextual congruence conditions: congruent and incongruent. During the congruence-judgment phase, old and similar objects were viewed along with congruent or incongruent context words (by condition). During the recognition 


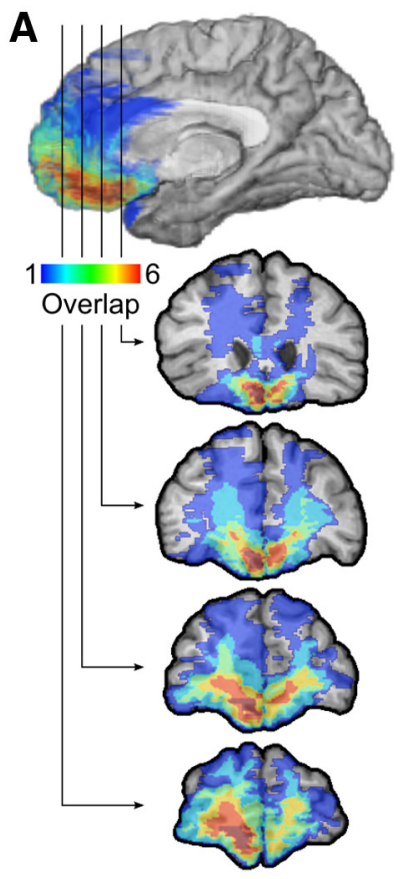

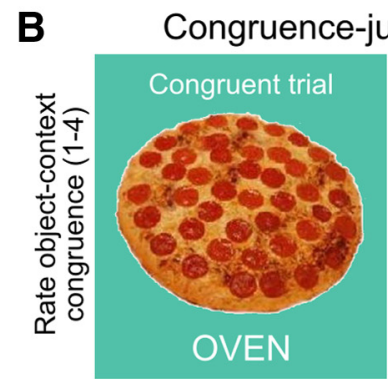

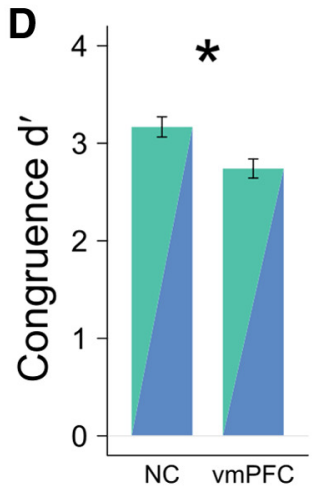

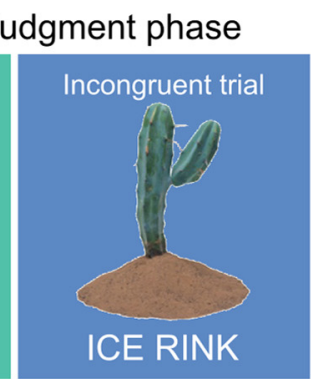
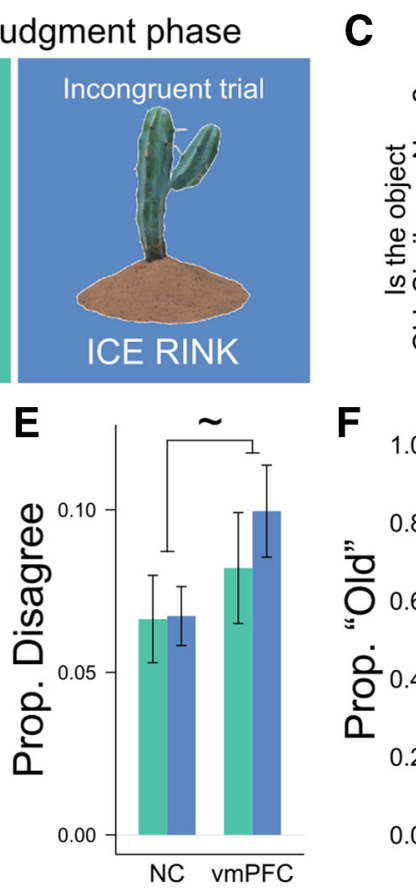

Figure 1. A, Neuroanatomy of the vmPFC group. Lesion overlap was concentrated in the vmPFC. Hotter colors indicate more cases with overlapping lesions (maximum of 6). $\boldsymbol{B}$, Congruencejudgment phase. Participants were asked to visualize items in congruent or incongruent contexts and then rate congruence. $C$, Recognition phase. Participants viewed each item and decided if it was old (left), similar (right), or new (condition not shown). In the similar condition, visually similar exemplars were substituted for studied objects. $D$, Congruence detection accuracy. Accuracy ( $d^{\prime}$ ) of the NC and vmPFC groups at identifying normative congruence in the congruence-judgment phase. The NC group was significantly more accurate. $\boldsymbol{E}$, Congruence-judgment disagreement. Mean proportion of items that produced disagreement with normative congruence. Disagreement was statistically symmetric across congruence conditions, and overall the vmPFC group disagreed marginally more often than the NC group. F, Schematic influence on recognition. The NC group showed an increased probability of identifying objects as "old" when visualized in congruent contexts; the vmPFC group did not. Bars show proportions derived from Model 1; * indicates significant difference between congruence conditions by linear contrast. Error bars in $\boldsymbol{D}$ and $\boldsymbol{E}$ indicate $\mathbf{S E M}$ and empirical $95 \%$ bootstrap confidence intervals in $\boldsymbol{F}$.

phase, stimuli included studied exemplars of old objects, nonstudied exemplars of similar objects, and intra-experimentally novel new objects. A relatively large number of recognition probes per condition (96) were included to support a logistic regression analysis.

\section{Procedure}

Congruence-judgment phase. Participants viewed images of objects (e.g., a cactus) for $4 \mathrm{~s}$ with context words that were congruent ("desert") or incongruent ("ice rink"; Fig. 1B) under these instructions: "Visualize a scene containing the specific pictured object in the accompanying setting." Two response prompts followed: "How clear was your mental image of the object in the context?" (1-4, low to high) and "How congruent are the object and context?" (1-4, low to high). Ten practice trials introduced this phase.

Recognition phase. Participants indicated whether each object was old, similar, or new and response confidence (1-4, low to high) with button presses (Fig. 1C). Response time (RT) was collected.

\section{Analysis}

For all tests, $\alpha=0.05$. Planned comparisons were nonpaired equalvariance $t$ tests.

Congruence-judgment phase. Subjective congruence per trial was operationalized as a dichotomous variable derived from congruence ratings (incongruent, 1|2; congruent, 3|4). A signal detection theory approach was used to measure subjective agreement with normative congruence $\left(d^{\prime}\right)$. An ANOVA was used to investigate group differences in the proportion of items producing disagreement between objective and subjective congruence and to examine congruent and incongruent conditions independently. A planned comparison evaluated group differences in visual imagery ratings.

Recognition phase. A planned comparison tested group differences in overall recognition performance using mean proportion correct for old and new objects.

Schematically congruent contexts were expected to modulate memory performance in the NC group, increasing the probability of identifying similar and old items as "old." Meanwhile, we hypothesized that vmPFC damage would reduce this influence of schematic memory. To investigate these group differences, we used a multilevel logistic regression model (LRM) to evaluate the fixed effects of group (vmPFC, NC) and congruence (congruent, incongruent) on the probability of calling items "old." Factors outside the focus of this investigation were controlled using a random-effects structure including random intercepts (for item and participant) and random slopes (per participant for recognition RT and confidence)

We compared LRMs using log likelihood ratio tests to identify the best-fit model (Table 2). The best model for these data (Model 1) was found to include group (NC, vmPFC), congruence (congruent, incongruent), item type (old, similar, new), the group $\times$ congruence interaction, normalized confidence and RT at during recognition, previous response one-back (i.e., preceding item called "old"), and previous response two-back. The group $\times$ congruence interaction was a key indicator of schematic influence of congruent context at the group level. Significance and variability of model terms was determined using bootstrap techniques robust to small sample sizes (Halekoh and Højsgaard, 2012).

To address potential group differences in subjective congruence ratings, another model (Model 2) replaced the normative item congruence in Model 1 with a subjective equivalent. Both models produced qualitatively similar results (Table 2).

\section{Results}

\section{Congruence-judgment phase}

Consistent with a role for the vmPFC in schematic processing, the vmPFC group rated item congruence in ways that were less aligned with normative expectations than the NC group $\left(t_{(16)}=\right.$ $2.62, p=0.019$; Fig. $1 D$ ). Similarly, the vmPFC group disagreed with normative object-context congruence categorization (i.e., 
Table 2. Recognition phase multilevel logistic regression model results

\begin{tabular}{|c|c|c|c|c|c|c|c|c|c|c|c|}
\hline \multirow[b]{2}{*}{ Effects } & \multirow[b]{2}{*}{ Description } & \multicolumn{5}{|c|}{ Model 1: normative congruence } & \multicolumn{5}{|c|}{ Model 2: subjective congruence } \\
\hline & & $\beta$ & SE & Zvalue & Sig. & $\mathrm{OR}$ & $\beta$ & SE & Zvalue & Sig. & OR \\
\hline Group & vmPFC or NC & -0.022 & 0.137 & -0.161 & & 0.96 & -0.020 & 0.137 & -0.143 & & 0.96 \\
\hline Object-context congruence & Congruent or incongruent & 0.136 & 0.147 & 2.893 & ** & 1.31 & 0.053 & 0.047 & 1.116 & & 1.11 \\
\hline Item type & Old, similar, or new & 0.811 & 0.062 & 13.185 & ** & 5.06 & 0.808 & 0.061 & 13.184 & *** & 5.03 \\
\hline Group $\times$ object- context congruence & Two-way interaction & 0.124 & 0.047 & 2.659 & * & 1.13 & 0.110 & 0.047 & 2.341 & * & 1.12 \\
\hline Group $\times$ item type & Two-way interaction & 0.040 & 0.047 & 0.855 & & 1.04 & 0.037 & 0.047 & 0.799 & & 1.04 \\
\hline Item type $\times$ object-context congruence & Two-way interaction & 0.008 & 0.047 & 0.180 & & 1.01 & 0.015 & 0.047 & 0.316 & & 1.02 \\
\hline Group $\times$ item type $\times$ object- context congruence & Three-way interaction & -0.017 & 0.046 & -0.363 & & 0.98 & 0.003 & 0.047 & 0.071 & & 1.00 \\
\hline Confidence at test (normalized) & Confidence rating (1-4) & 0.976 & 0.218 & 4.469 & $* * *$ & 2.65 & 0.977 & 0.219 & 4.466 & $* * *$ & 2.66 \\
\hline Response time at test (normalized) & Response time & -0.549 & 0.104 & -5.252 & $* * *$ & 0.58 & -0.547 & 0.105 & -5.203 & $* * *$ & 0.58 \\
\hline Previous response (one-back) & Resp. to prev. item & 0.185 & 0.092 & 2.017 & * & 1.20 & 0.180 & 0.091 & 1.973 & * & 1.20 \\
\hline Previous response (two-back) & Resp. to item before prev. & 0.188 & 0.091 & 2.070 & * & 1.21 & 0.180 & 0.091 & 1.992 & * & 1.20 \\
\hline
\end{tabular}

The best-fit model (Model 1) and an alternative model (Model 2) analyzing recognition-phase "old" responses. The group $\times$ congruence effect was the key test of differential influence of previous context on recognition and was statistically significant in both models. Model 1 included normative congruence categories as a predictor. Model 2 substituted subjective congruence categories. $\beta$, Coefficient; $0 \mathrm{R}$, odds ratio; Sig., statistical significance of predictor. ${ }^{*} p<0.05$, ${ }^{* *} p<$ $0.01,{ }^{* * *} p<0.001$.

reported congruent items as incongruent and vice versa; Fig. $1 E)$ marginally more often than the NC group $\left(F_{(1,36)}=3.112, p=\right.$ 0.087 ; note that rates of disagreement did not differ by condition for either group, each $t<0.7$, each $p>0.5)$. Although there were significant group differences in congruence ratings, overall both groups agreed with normative schematic congruence on $>90 \%$ of trials. Importantly, visual imagery ratings did not differ between groups $\left(t_{(16)}=0.97, p=0.348\right)$.

\section{Recognition phase}

Recognition accuracy: planned comparison

The vmPFC and NC groups did not differ in recognition accuracy for old and new objects $\left(t_{(16)}=0.20, p=0.844\right)$, indicating that the vmPFC group did not have impaired recognition (corroborated by neuropsychological findings; Table 1).

\section{Logistic regression}

For the NC group, but not the vmPFC group, schematic memory was predicted to lead to an increased probability of objects studied in the congruent condition being identified as "old." This effect was predicted to generalize across old and similar objects. Model results are displayed in Table 2 and Figure $1 F$. There was a reliable main effect of congruence such that objects presented in a congruent context were more likely to be called "old" ( $p=$ $0.004)$. However, as predicted, group significantly modulated this effect as evidenced by a significant group $\times$ congruence interaction $(p=0.011)$. Consistent with our hypothesis, the NC group was more likely to call objects "old" when they were paired with a congruent rather than incongruent context, whereas the vmPFC group did not show this effect. Linear contrasts indicated that, for the NC group, this schematic influence was present for similar items (i.e., false recognition, $p=0.003$ ) and old items (i.e., true recognition, $p=0.044$ ). However, for the vmPFC group, there was not a significant effect of congruence for either old or similar items ( $p=0.679$ and $p=0.517$, respectively). Additionally, there was a significant main effect of object type, indicating that old items were more likely than similar items to be identified as "old" ( $p=0.001)$.

Because vmPFC participants were more likely to disagree with normative object-context congruence, a second model (Model 2) was fit using subjective rather than normative congruence in Model 1 (see above, Analysis; Table 2). Model 2 results were consistent with Model 1 . Critically, a significant group $\times$ congruence interaction was observed, which indicated that NC participants were more likely to identify objects as "old" when paired with a subjectively congruent rather than incongruent context while vmPFC participants did not show this effect. Consistent with Model 1 findings, linear contrasts indicated that the NC group showed a significant effect of congruence for both old and similar items ( $p=0.032$ and $p=0.044$, respectively), whereas the vmPFC group showed neither effect $(p=0.679$ and $p=0.517$, respectively). Therefore, findings from Model 2 were qualitatively similar to Model 1, indicating that the difference in schematic influence between groups was not attributable to a difference in subjective congruence.

\section{Discussion}

Schematic memory allows for generalization from previous experience, but this frequently advantageous process has been hypothesized to produce memory errors by reducing accuracy for details of schematically congruent experiences. In this study, schematic influence was operationalized as the tendency to recognize an item as old when previous exposure was in a schematically congruent context. We found that prior congruence modulated recognition in healthy participants, but the effect was reduced for vmPFC participants in both advantageous and disadvantageous circumstances. These results extend previous findings from animal models (Euston and McNaughton, 2006; Tse et al., 2007), human functional neuroimaging (Kumaran et al., 2009; van Kesteren et al., 2013; Zeithamova et al., 2012a,b), and earlier neuropsychological studies (Ghosh et al., 2014; Warren et al., 2014). The reduced schematic influence of congruence for the vmPFC group suggests an important role for the vmPFC in integrating previous experience into ongoing memory processes.

The attenuation of schematic influence in participants with vmPFC damage was evident for recognition of both old and similar objects. This reduction of schematic influence for both veridical and false memory was a key finding consistent with our proposed role of vmPFC as necessary for detecting and using schematic congruence (Warren et al., 2014). Meanwhile, the NC group was more likely to accurately recognize old items visualized in a congruent context, implying that schematic memory conferred a congruence advantage in this condition. This parallels evidence indicating that schematic congruence can facilitate learning and memory (Morris, 2006; Tse et al., 2007). In addition, the NC group was biased to falsely recognize lure objects similar to those visualized in congruent contexts, suggesting a congruence disadvantage in this condition. One interpretation is that NC participants encoded a generalized memory of schematically congruent items during initial exposure and that this generalized 
memory sometimes lacked the specificity needed to reject similar objects during recognition.

Critically, our study showed that advantageous and disadvantageous effects of schematic memory were reduced in participants with vmPFC damage, which is consistent with the proposition that the vmPFC group formed memories of similar detail regardless of contextual congruence. Our theory-driven account (van Kesteren et al. 2012) of vmPFC-dependent changes in representational specificity remains speculative. Future investigations could directly test this prediction by collecting functional neuroimaging data during a similar task and then conducting multivoxel pattern analysis to evaluate congruencerelated representational quality in hippocampus and vmPFC.

Participants with vmPFC damage and NC participants showed comparable levels of accuracy for old and new stimuli, indicating that the group difference in false memory performance was not attributable to an overall memory deficit. This aligns with previous work demonstrating that broader PFC damage is not associated with profound declarative memory deficits (Moscovitch and Winocur, 1995) but that PFC damage can disrupt application of schematic memory, for example, within a categorized list (della Rocchetta and Milner, 1993; Gershberg and Shimamura, 1995).

vmPFC influences on schematic memory were observed during the congruence-judgment phase of the experiment as well. Interestingly, participants with vmPFC damage were significantly more likely to disagree with normative object-context congruence (i.e., categorize a congruent pairing as incongruent or vice versa) than NC participants. This is consistent with a role for the vmPFC in detecting congruence online in addition to encoding schematically congruent memories. Notably, group differences in online schematic influence did not account for differences in the schematic influence of context on recognition (Table 2, Model 2).

Evidence of reduced schematic influence in the vmPFC group suggests that the vmPFC plays a key role in a network of brain regions supporting schematic memory. Neuroimaging studies have shown that schema acquisition increases activation of both the hippocampus and the vmPFC, indicating that both regions are correlated with schematic encoding (Kumaran et al. 2009, Zeithamova et al. 2012b). Additional evidence has shown that the MPFC and medial temporal lobe (MTL) are functionally connected (Hyman et al., 2010) and that the vmPFC may directly influence memory formation via functional coupling with the MTL (Ranganath et al., 2005). Functional connections between the vmPFC and MTL are consistent with well characterized monosynaptic connections between the regions (Cavada et al., 2000; Saleem et al., 2008).

Previous work suggests that the vmPFC has a reciprocal relationship with the MTL. van Kesteren et al. (2012) proposed that experiencing schema-consistent information engages a schematic memory system, including the vmPFC, causing generalization of the resulting memory. When schematic consistency is absent, the MTL and hippocampus encode a detailed episodic memory (van Kesteren et al., 2012). The findings of the present study are consistent with this proposal because the reduced schematic influence observed in the vmPFC group may have led to the formation of specific, detailed memories of objects in both congruent and incongruent contexts. Conversely, future work could evaluate whether MTL damage promotes generalized schematic memories. Such findings could have implications for healthy populations, potentially addressing the increased reliance on schematic memory observed in older adults that may be related to age-related difficulty with associative memory (Castel et al.,
2013). In support of this, much of the cerebral cortex atrophies with age, but while the decline seen in the MPFC follows a gradual, lifelong trajectory, hippocampal atrophy accelerates in old age (Fjell et al. 2013). This volumetric dissociation over the lifespan may promote reliance on the vmPFC-schematic memory system in older adults and increase potential real-world vulnerabilities to false recognition or deceptive advertising (Denburg et al., 2007).

Although our findings were statistically robust and derived from many observations of each participant, our study had some limitations. As in many neuropsychological investigations, our sample size was relatively small. The limited sample resulted from the inclusion of only vmPFC participants with stable, focal, bilateral lesions and neuropsychologically normal memory. Also, the current study used a novel implementation of a previously described paradigm (van Kesteren et al., 2012), although pilot and normative data (neither reported here) were used to refine the procedure. These qualifications are important, but any limits they placed on statistical power in our study were insufficient to obscure the effects of interest.

In conclusion, the vmPFC has long been postulated to play a key role in the integration of environmental, physiological, and mnemonic signals to influence complex decision-making (Bechara and Damasio, 2005). The present findings suggest that the vmPFC additionally plays a key role in integrating previous experiences and contextual information with current experience to form schematic memories. This role in memory is consistent with the proposition that the vmPFC is part of a network of brain regions, including the MTL, that support declarative memory processes. As an important component process of declarative memory, schematic memory facilitates the representation of complex concepts, influences the encoding of new material, and guides complex behaviors (Bartlett, 1932). In our study, vmPFC damage reduced the influence of schematic memory, whether advantageous (during true recognition) or disadvantageous (during false recognition). Our findings extend previous work from functional neuroimaging and neuropsychological investigations by showing that the vmPFC is necessary for normal schematic influence on recognition. Furthermore, our results suggest that the vmPFC plays a key role in detecting and incorporating contextual congruence, potentially improving our ability to distinguish the surreal from the banal. These findings enhance our knowledge of the neural correlates of schematic memory and advance our understanding of the brain networks supporting memory processes.

\section{References}

Bartlett F (1932) Remembering. Cambridge: Cambridge UP.

Bechara A, Damasio AR (2005) The somatic marker hypothesis: a neural theory of economic decision. Games Econ Behav 52:336-372. CrossRef

Bonnici HM, Chadwick MJ, Lutti A, Hassabis D, Weiskopf N, Maguire EA (2012) Detecting representations of recent and remote autobiographical memories in vmPFC and hippocampus. J Neurosci 32:16982-16991. CrossRef Medline

Castel AD, McGillivray S, Worden KM (2013) Back to the future: past and future era-based schematic support and associative memory for prices in younger and older adults. Psychol Aging 28:996-1003. CrossRef Medline

Cavada C, Compañy T, Tejedor J, Cruz-Rizzolo RJ, Reinoso-Suárez F (2000) The anatomical connections of the macaque monkey orbitofrontal cortex: a review. Cereb Cortex 10:220-242. CrossRef Medline

della Rocchetta AI, Milner B (1993) Strategic search and retrieval inhibition: the role of the frontal lobes. Neuropsychologia 31:501-524.

Denburg NL, Cole CA, Hernandez M, Yamada TH, Tranel D, Bechara A, Wallace RB (2007) The orbitofrontal cortex, real-world decision making, and normal aging. Ann NY Acad Sci 1121:480-498. CrossRef Medline 
Euston DR, McNaughton BL (2006) Apparent encoding of sequential context in rat medial prefrontal cortex is accounted for by behavioral variability. J Neurosci 26: 13143-13155. CrossRef Medline

Fjell AM, Westlye LT, Grydeland H, Amlien I, Espeseth T, Reinvang I, Raz N, Holland D, Dale AM, Walhovd KB (2013) Critical ages in the life course of the adult brain: nonlinear subcortical aging. Neurobiol Aging 34: 2239-2247. CrossRef Medline

Frank RJ, Damasio H, Grabowski TJ (1997) Brainvox: an interactive, multimodal visualization and analysis system for neuroanatomical imaging. Neuroimage 5:13-30. CrossRef Medline

Gershberg FB, Shimamura AP (1995) Impaired use of organizational strategies in free recall following frontal lobe damage. Neuropsychologia 33: 1305-1333. CrossRef Medline

Ghosh VE, Moscovitch M, Melo Colella B, Gilboa A (2014) . Schema representation in patients with ventromedial PFC lesions. J Neurosci 34: 12057-12070. CrossRef Medline

Halekoh U, Højsgaard S (2012) A Kenward-Roger approximation and parametric bootstrap methods for tests in linear mixed modelsthe R package pbkrtest. J Stat Softw 59. Retrieved from http://people. math.aau.dk/ sorenh/software/pbkrtest/doc/pbkrtest-JSS.pdf.

Hyman JM, Zilli EA, Paley AM, Hasselmo ME (2010) Working memory performance correlates with prefrontal-hippocampal theta interactions but not with prefrontal neuron firing rates. Front Integr Neurosci 4:2. CrossRef Medline

Koriat A, Goldsmith M, Pansky A (2000) Toward a psychology of memory accuracy. Annu Rev Psychol 51:481-537. Medline

Kumaran D, Summerfield JJ, Hassabis D, Maguire EA (2009) Tracking the emergence of conceptual knowledge during human decision making. Neuron 63:889-901. CrossRef Medline

Kumaran D, Warren DE, Tranel D (2015) Damage to the ventromedial prefrontal cortex impairs learning from observed outcomes. Cereb Cortex.

Lewis PA, Durrant SJ (2011) Overlapping memory replay during sleep builds cognitive schemata. Trends Cogn Sci 15:343-351. CrossRef Medline

Melo B, Winocur G, Moscovitch M (1999) False recall and false recognition: an examination of the effects of selective and combined lesions to the medial temporal lobe/diencephalon and frontal lobe structures. Cogn Neuropsychol 16: 343-359. CrossRef

Morris RG (2006) Elements of a neurobiological theory of hippocampal function: the role of synaptic plasticity, synaptic tagging and schemas. Eur J Neurosci 23:2829-2846. CrossRef Medline
Moscovitch M, Winocur G (1995) Frontal lobes, memory, and aging. Ann NY Acad Sci 769:119-150. CrossRef Medline

Palmer TE (1975) The effects of contextual scenes on the identification of objects. Mem Cognit 3:519-526. CrossRef Medline

Ranganath C, Heller A, Cohen MX, Brozinsky CJ, Rissman J (2005) Functional connectivity with the hippocampus during successful memory formation. Hippocampus 15:997-1005. CrossRef Medline

Saleem KS, Kondo H, Price JL (2008) Complementary circuits connecting the orbital and medial prefrontal networks with the temporal, insular, and opercular cortex in the macaque. J Comp Neurol 506:659-693. CrossRef Medline

Tse D, Langston RF, Kakeyama M, Bethus I, Spooner PA, Wood ER, Witter MP, Morris RG (2007) Schemas and memory consolidation. Science 316:76-82. CrossRef Medline

van Kesteren MT, Rijpkema M, Ruiter DJ, Fernández G (2010) Retrieval of associative information congruent with prior knowledge is related to increased medial prefrontal activity and connectivity. J Neurosci 30: 15888-15894. CrossRef Medline

van Kesteren MT, Ruiter DJ, Fernández G, Henson RN (2012) How schema and novelty augment memory formation. Trends Neurosci 35: 211-219. CrossRef Medline

van Kesteren MT, Beul SF, Takashima A, Henson RN, Ruiter DJ, Fernández G (2013) Differential roles for medial prefrontal and medial temporal cortices in schema-dependent encoding: from congruent to incongruent. Neuropsychologia 51:2352-2359. CrossRef Medline

Wang SH, Morris RG (2010) Hippocampal-neocortical interactions in memory formation, consolidation, and reconsolidation. Annu Rev Psychol 61:49-79, C1-4. CrossRef Medline

Warren DE, Jones SH, Duff MC, Tranel D (2014) False recall is reduced by damage to the ventromedial prefrontal cortex: implications for understanding the neural correlates of schematic memory. J Neurosci 34: 7677-7682. CrossRef Medline

Yassa MA, Lacy JW, Stark SM, Albert MS, Gallagher M, Stark CE (2011) Pattern separation deficits associated with increased hippocampal CA3 and dentate gyrus activity in nondemented older adults. Hippocampus 21:968-979. CrossRef Medline

Zeithamova D, Schlichting ML, Preston AR (2012a) The hippocampus and inferential reasoning: building memories to navigate future decisions. Front Hum Neurosci 6:70. CrossRef Medline

Zeithamova D, Dominick AL, Preston AR (2012b) Hippocampal and ventral medial prefrontal activation during retrieval-mediated learning supports novel inference. Neuron 75:168-179. CrossRef Medline 